\title{
Wound-Healing with Mechanically Robust and Biodegradable Hydrogel Fibers Loaded with Silver Nanoparticles
}

\author{
Kevin Neibert, Venkateshwarlu Gopishetty, Anton Grigoryev, Ihor Tokarev, Noura Al-Hajaj, \\ Joshua Vorstenbosch, Anie Philip, Sergiy Minko,* and Dusica Maysinger**
}

The objective of this study is to provide a novel synthetic approach for the manufacture of wound-healing materials using covalently cross-linked alginate fibers loaded with silver nanoparticles. Alginate fibers are prepared by wet-spinning in a $\mathrm{CaCl}_{2}$ precipitation bath. Using this same approach, calcium cross-links in alginate fibers are replaced by chemical cross-links that involve hydroxyl groups for subsequent cross-linking by glutaraldehyde. The cross-linked fibers become highly swollen in aqueous solution due to the presence of carboxyl functional groups, and retain their mechanical stability in physiological fluids owing to the stabilized network of covalent bonds. Alginate fibers can then be loaded with silver ions via the ion-exchange reaction. Silver ions are reduced to yield $11 \mathrm{~nm}$ silver nanoparticles incorporated in the polymer gel. This method provides a convenient platform to incorporate silver nanoparticles into alginate fibers in controlled concentrations while retaining the mechanical and swelling properties of the alginate fibers. Our study suggests that the silver nanoparticles loaded fibers may be easily applied in a wound healing paradigm and promote the repair process though the promotion of fibroblast migration to the wound area, reduction of the inflammatory phase, and the increased epidermal thickness in the repaired wound area, thereby improving the overall quality and speed of healing.

phases: i) inflammation, ii) tissue formation and iii) tissue remodelling. ${ }^{[1]}$ The inflammatory phase is marked by an infiltration of neutrophils, monocytes and macrophages, which results in the clearance of bacteria and tissue debris from the wound area. The tissue formation phase involves the coordinated migration of vascular endothelial cells and fibroblasts into the wound area, and deposition of a provisional extracellular matrix. In the remodelling phase, constituent cells synthesize and degrade extracellular matrix proteins and differentiated myofibroblasts mediate contraction of the wound. ${ }^{[2]}$ A host of modern approaches have been developed for wound management, which aim to facilitate the natural wound healing process or restore healing which may be impaired due to pathological conditions.

A number of different wound dressing materials have been explored in the last 20 years to prevent wound infection. ${ }^{[3]}$ Alginate is a naturally occurring, water soluble polysaccharide, extracted from brown marine algae and is used in a wide

\section{Introduction}

Wound healing is a highly dynamic and complex physiological process that involves a series of well-orchestrated cellular and molecular events. It is characterized by three overlapping

K. Neibert, N. Al-Hajaj, D. Maysinger Department of Pharmacology and Therapeutics McGill University

Montreal, Quebec, Canada

E-mail: Dusica.maysinger@mcgill.ca

V. Gopishetty, A. Grigoryev, I. Tokarev, S. Minko

Department of Chemistry and Bimolecular Science

Clarkson University

New York, USA

E-mail:sminko@clarkson.edu

J. Vorstenbosch, A. Philip

Department of Surgery

McGill University

Montreal, Quebec, Canada

DOI: 10.1002/adhm.201200075 range of applications, including biomedical products and the pharmaceutical industry. ${ }^{[4]}$ Among the numerous varieties of polysaccharides, alginate has a number of favourable physiochemical properties including: highly absorbent, biocompatible, non-allergic, bacteriostatic, fungistatic and haemostatic, which are attractive for wound healing applications. ${ }^{[8-11]}$ Alginate is capable of absorbing large volumes of water, forming highly stable hydrogels. ${ }^{[12]}$ As the alginate swells, incorporated drugs can be released from the alginate matrix. Alginate fibers have been processed into woven, knitted and composite materials and may be supplemented with additional cargo, forming stable, controlled-release wound dressings that provide complementary wound management functionality. ${ }^{[13]}$ Dry alginate dressings stably adhere to the wound area through mucoadhesive contacts. As alginate comes in contact with body fluids, the dry fibers transform into a slippery hydrogel, effectively separating the wound from the surrounding tissues and forming a tissue adhesion barrier. ${ }^{[14]}$ As the wound dressing absorbs large volumes of exudate, the spaces between the fibers close, trapping the exudate within the dressing matrix, 
increasing bacterial retention, while maintaining a moist environment required for efficient wound healing. ${ }^{15]}$ In addition to traditional alginate-based wound dressings, alginate microspheres and hydrogels have been used as delivery vehicles for drugs that promote wound healing. ${ }^{[16-19]}$

In recent years, silver has gained prominence in wound management as a number of clinically relevant silver-containing wound dressings have been developed. ${ }^{[20-22]}$ Silver-containing materials have a long history as anti-microbial agents, especially in the treatment of wounds and burns. ${ }^{[23]}$ In fact, silver sulfadiazine is considered to be the gold standard in the topical treatment of burns. ${ }^{[24]}$ There is a renewed interest in silver based anti-bacterials provoked by the emergence of highly resistant bacteria and the increasing prevalence of hospital-acquired bacterial infections. Silver ions $\left(\mathrm{Ag}^{+}\right)$are effective against a wide range of bacteria, including highly resistant strains such as meticillin-resistant staphylococcus aureus (MRSA). ${ }^{[25,26]}$ Although silver is a highly effective anti-microbial agent, its use has been limited due to observed toxicity in mammalian cells. ${ }^{[27,28]}$ One way to overcome this problem is to construct a system which allows the controlled release of silver ions, promoting wound healing, while minimising toxicity. ${ }^{[29]}$ Loading silver nanoparticles into alginate fibers is an attractive approach to improve the silver carrying capacity of the fibers while retaining their favourable mechanical properties and stability.

Due to the toxicity associated with silver ions, alternative silver formulations and delivery systems are being investigated. The small size of silver nanoparticles (nanocrystals) has been correlated to an increased efficacy against bacteria and more importantly, lower toxicity in humans, compared to other silver containing compounds (ie. silver nitrate). ${ }^{[30]}$ In addition to the antibacterial effect of silver ions being released from nanoparticles, recent evidence suggests that silver nanoparticles exert potent anti-inflammatory effects which accelerates wound healing, although the mechanism of action is not well known. ${ }^{[27,31,32]}$ Silver nanoparticles can be embedded into a wide verity of materials and fabrics ranging from socks to wound dressings. In order to incorporate silver ions into alginate materials, calcium alginate fibers are treated with aqueous solutions of silver nitrate $\left(\mathrm{AgNO}_{3}\right)$. The silver ions in the solution exchange with calcium ions in the fiber, resulting in the formation of calcium alginate fiber containing silver ions. ${ }^{[33]}$ The drawback of this method is that the same carboxylic groups are used for cross-linking with calcium ions and for binding silver ions. In calcium cross-linked fibers, an increase in the $\mathrm{Ag}^{+}$-ion content implies a decrease in a fraction of $\mathrm{Ca}^{2+}$-crosslinks which are replaced by monovalent silver ions and, in turn, the deterioration of mechanical properties and stability of the fibers.

In this article, we report a novel synthesis of alginate fibers loaded with silver nanoparticles and their application for wound dressing in series of in vitro experiments with cells and in vivo incisional wound model using experimental animals (mice). We overcome the aforementioned trade-off between the capacity of alginate fibers for silver and their mechanical stability by decoupling the processes of cross-linking and loading of the material with silver. In our approach, alginate fibers are prepared by a well-established wet-spinning method using a cross-linking bath containing calcium ions (Supporting Figure S1). Calcium cross-links in alginate fibers are then replaced by chemical cross-links that involve hydroxyl groups for cross-linking by glutaraldehyde. The alginate fibers loaded with silver ions are treated in reduction bath to yield alginate fibers that carry silver nanoparticles. Cross-linked carboxylic functional groups provide a very high swelling degree of the alginate fibers (up to 2000\%) in physiological fluids. Consequently, the proposed approach combines the facile wet-spinning method and chemical crosslinking protocol to produce robust fibers with superabsorbent properties and the ability to vary the amount of silver in the fibers.

\section{Experimental Section}

\subsection{Animals}

SKH-1 hairless mice were obtained from Charles River Laboratories. The mice were housed five per cage under constant humidity and temperature with $12 \mathrm{~h}$ light/ dark cycles. They were allowed access to water and standard mouse feed ad libitum and were monitored daily. Healthy, six/seven week old mice, weighing between 23 and 26 g were selected for experimental work. All experimental procedures received approval by the McGill Animal Care Facility. The SKH-1 mice possess a splicing defect in the hair root gene that causes apoptosis, resulting in alopecia after the first hair cycle. Also, the epidermis of SKH-1 mice is thicker than that of other mouse strains, making it an attractive model for wound healing of human skin. No immune abnormalities have been reported. ${ }^{[34]}$

\subsection{Wet Spinning of Alginate Fibers}

Alginate fibers were prepared by a wet-spinning method. Medium viscosity (2000 cps for $2 \%$ solution at $25^{\circ} \mathrm{C}$ ) sodium alginate from brown algae (Sigma) was dissolved in Millipore water at $60{ }^{\circ} \mathrm{C}$ while stirring to form a $1.5 \mathrm{wt} . \%$ solution. The resulting spinning solution was allowed to stay without stirring for $10 \mathrm{~min}$ to ensure that all the entrapped air bubbles were released. The spinning was carried out in an apparatus that consisted of a syringe pump set in the vertical syringe position and a coagulation bath placed on an orbital shaker (VWR, Model DS-500). The alginate solution was injected at the constant rate of $2 \mathrm{ml} / \mathrm{min}$ through a 20 -gauge pipetting needle, acting as a spinneret, into the coagulation bath containing $1 \mathrm{M}$ $\mathrm{CaCl}_{2}$ aqueous solution. Calcium ions caused the immediate gelation of alginate which formed a continuous fiber coming out at the tip of the needle. The fiber was collected as a loose coil at the bottom of the coagulation bath that moved along the circular path in the horizontal plane due to the rotary shaker. Such circular motion occurring at the frequency of $0.6 \mathrm{~Hz}$ and the circle radius of $1 \mathrm{~cm}$ provided some fiber elongation and ensured the easy fiber disentanglement later on. The fiber coil was kept in the coagulation bath for at least $30 \mathrm{~min}$ to provide sufficient cross-linking time and then transferred into a $0.2 \mathrm{M}$ $\mathrm{CaCl}_{2}$ aqueous solution for further cross-linking overnight. The ionically cross-linked alginate fiber was washed three times in Millipore water and dried in air. 


\subsection{Chemical Cross-Linking of Alginate Fibers}

To ensure the stability of alginate fibers in a wide range of environmental conditions, they were chemically cross-linked via hydroxyl groups. ${ }^{[35]}$ To this end, fiber samples were placed in a water-acetone mixture $(1: 7.5 \mathrm{v} / \mathrm{v})$ containing $0.36 \mathrm{M}$ glutaraldehyde and $50 \mathrm{mM} \mathrm{HCl}$ for $48 \mathrm{~h}$ at $40{ }^{\circ} \mathrm{C}$. In this cross-linking method, hydroxyl groups of alginate reacted with aldehyde groups of glutaraldehyde while the acid acted as a catalyst of the acetalization reaction. Afterwards, the fibers were removed from the reaction mixture and washed three times in water.

\subsection{Synthesis of Silver Nanoparticles in the Alginate Fibers}

The synthetic protocol included the following steps. In the first step, silver ions were loaded into fibers by ion exchange from a silver nitrate solution ( 0.05 or $0.005 \mathrm{mM}$ ) for $1 \mathrm{~h}$. Afterwards the fiber sample was removed and the excess of the $\mathrm{AgNO}_{3}$ solution was removed by soaking the fibers with KimWipes. In the second step, the sample was transferred into a sodium borohydride solution for $10 \mathrm{~min}$ to reduce the silver ions into metallic silver. The concentration of the reducing agent was $0.07 \mathrm{mM}$ in the case of the fibers treated with the $0.05 \mathrm{mM} \mathrm{AgNO}_{3}$ solution and $0.007 \mathrm{mM}$ in the case of the fibers treated with the $0.005 \mathrm{mM} \mathrm{AgNO}_{3}$ solution. After the reduction step, the fibers were washed three times in water and dried in air.

\subsection{Synthesis of Reference Samples of Silver Nanoparticles in Aqueous Solution}

A dispersion of $11 \mathrm{~nm}$ in diameter silver nanoparticles was obtained by mixing $0.07 \mathrm{mM}$ sodium borohydride and $0.05 \mathrm{mM}$ $\mathrm{AgNO}_{3}$ aqueous solutions at ambient temperature and purified by dialysis.

\subsection{Characterization of the Fibers and Silver Nanoparticles}

Alginate fibers and silver nanoparticles (AgNPs) were characterized by scanning electron microscopy (SEM), energy-dispersive X-ray spectroscopy (SEM-EDS), atomic absorption spectrometry (AAS), transmission electron microscopy (TEM), atomic force microscopy (AFM), UV-vis spectroscopy, and Fourier transform infrared (FTIR) spectroscopy. In particular, the surface morphology of alginate fiber samples was visualized with a JEOL 7400 high resolution field emission scanning electron microscope (JEOL Ltd). The amount of silver reduced in the alginate fibers was analyzed with Perkin Elmer Flame Atomic Absorption Spectrometer (model 3100). The SEM-EDS was used to assess the amount of the reduced silver on the fiber surface and cross-sectional planes of cryogenically fractured fibers. Silver nanoparticles reduced in the fibers were visualized using a Jeol JEM 2010 high-resolution transmission electron microscope (JEOL) and a Dimension 3100 microscope (Veeco Instruments) operating in the tapping mode. Prior to the measurements, the fibers were enzymatically degraded with alginate lyase, and silver nanoparticles released from the fiber inte- rior were deposited onto TEM grids or silicon substrates. The adsorption spectra of the ionically and chemically cross-linked alginate fibers were recorded on a single-beam microprocessorcontrolled diode array spectrophotometer with collimating optics (Hewlett-Packard, 8452A).

\subsection{Cell Culture and Treatments}

Mouse fibroblast (NIH/3T3) cells (ATCC, CRL- 2795) were seeded in Dulbecco's modified eagle's medium (DMEM, Gibco) containing $10 \%$ of bovine calf serum (Gibco) and $1 \%$ penicillin-streptomycin (Gibco). Cells were seeded in 12-well plates (Millipore) at a density of $2 \times 10^{5}$ cells/well maintained at $37{ }^{\circ} \mathrm{C}, 5 \% \mathrm{CO}_{2}$ in a humidified atmosphere. Cells were grown in serum containing media for 24 hours before cell treatments to attain confluency. Culture media was then aspirated, cells were washed with phosphate buffered saline (PBS, Gibco) and incubated at $37{ }^{\circ} \mathrm{C}$ with silver nanoparticles $(0-5 \mu \mathrm{M})$, basic fibroblast growth factor (FGF; Gibco, $0.291 \mu \mathrm{M}$ ) and mitomycin C (MitC; Sigma, $15 \mu \mathrm{M}$ ) for the times indicated (24-48 hours).

\subsection{MTT Cell Viability Assay}

Colorimetric MTT (3-(4,5-dimethylthiazol-2-yl)-2,5-diphenyl tetrazolium bromide, Sigma) assays were performed to assess the mitochondrial metabolic activity, an indirect measure of cell viability. ${ }^{[36]}$ Following treatment, media was removed and replaced with serum-free media containing MTT $(0.5 \mathrm{mg} / \mathrm{mL})$ and cells were then incubated for one hour at $37{ }^{\circ} \mathrm{C}$. Following the incubation, media was removed, cells were lysed and formazan was dissolved with dimethyl-sulfoxide (DMSO, Sigma). Absorbance was measured at $595 \mathrm{~nm}$ using a Benchmark microplate reader (Bio-Rad). All the measurements were done in triplicates in three or more independent experiments.

\subsection{Scratch Assay}

Once confluent, the cell monolayer was scratched in a straight line using $100 \mu \mathrm{l}$ pipette tip to mimic an incisional wound. Cells were then washed with PBS to remove cell debris. Fresh serum containing media was added and cells were treated with (i) silver nanoparticles $(0.31,0.625,1.2,2.5$ and $5 \mu \mathrm{M}$ with respect to silver concentration), ii) FGF $(0.291 \mu \mathrm{M})$ or iii) MitC $(15 \mu \mathrm{M})$ and incubated at $37^{\circ} \mathrm{C}$ for 24 or 48 hours, then cells were washed once with PBS and fixed with paraformaldehyde (4\%) (Fisher) for $15 \mathrm{~min}$ at room temperature (RT). Cells were then stained with Hoechst (Molecular probes; 33258, $10 \mu \mathrm{M}$ ) for $10 \mathrm{~min}$ at RT. Fluorescent images of cells were acquired at 20× with a Leica DFC350FX monochrome digital camera connected to a Leica DMI4000B inverted fluorescence microscope. Migrated cells were then counted using Image J software.

\subsection{Incisional Wound}

The incision wound model was used according to Ehrlich and Hunt. ${ }^{[3]}$ Equal number of hairless SKH-1 mice were divided 
into five groups and kept in individual cages: group 1, serving as control, received no treatment following the creation of the wounds, group 2 received unloaded alginate fiber, group 3 received the AgNP loaded alginate fiber $(0.05 \mathrm{mM} \mathrm{AgNO}$ ), group 4 received the AgNP loaded alginate fiber $(0.005 \mathrm{mM}$ $\mathrm{AgNO}_{3}$ ). The wound area in animals from group 5 was treated with nanosilver particles $(\mathrm{d}=11 \mathrm{~nm})$ in dispersion so that the concentration of silver (1.46 ppm Ag, $1.35 \mu \mathrm{M} \mathrm{Ag}, 0.187 \mathrm{nM}$ particles) was equal to that present in the nanosilver loaded alginate $\left(0.05 \mathrm{mM} \mathrm{AgNO}_{3}\right)$ fibers. Mice were anaesthetized prior to and during creation of the wound with $2 \%$ isoflurane. Dorsal $2.0 \mathrm{~cm}$ incisional wounds, one left lateral, one right lateral, were introduced through the skin surgically. After the incision, nanosilver loaded alginate fibers and unloaded fibers were embedded into the wound area in equal weight $(0.0012 \pm .0001$ g) and then sutured shut using sutures (Ethicon, 4-0 Nylon). Following surgery, the mice were wrapped with tegaderm (3M) and hyperfix (Delasco) to cover and protect the wound area. Animals were sacrificed on postoperative day 2 and 10 and wound tissue was excised and fixed in formalin for immunohistochemical and histological evaluation.

\subsection{Immunohistochemistry}

Rehydrated, serial paraffin sections $(5 \mu \mathrm{m})$ were immersed in Proteinase K stock solution (Sigma) to reactivate hidden or masked epitopes. Sections were washed with PBS and endogenous peroxidase activity blocked with methanol containing $0.3 \%$ hydrogen peroxide. Samples were then blocked with $5 \%$ normal goat serum (NGS, Vector Labs) followed by incubation with rat anti-mouse F4-80 (E bioscience) $1: 25$ in 5\% NGS and tris-buffered saline and tween 20 (TBST) or ly6-gr rat antimouse (E bioscience) 1:25 in 5\% NGS and TBST at $4{ }^{\circ} \mathrm{C}$ labeling macrophages and neutrophils respectively. Slides were rinsed with TBST and then incubated with biotinylated anti-rat secondary antibody (Vector Lab). The slides were washed with TBST and avidin-biotin complex (Vector Lab, ABC Reagent) added. The slides were rinsed in TBST, developed with chromagen 3,3'-diaminobenzidine (Sigma), and lightly stained with hematoxylin. The number of F4-80 and ly6-gr positive cells per high-power field in the wound base was then determined using ImageProPlus6 software.

\subsection{Histology}

Formalin-fixed skin grafts were bisected and paraffin embedded. Serial $5-\mu \mathrm{m}$ sections were obtained from each of the paraffinembedded wounds using a 30/50 microtome (Leica). Sections were collected on Superfrost Plus slides (Fisher Scientific) and stained with hematoxylin and eosin stain (Sigma) according to the protocol provided from the company.

\subsection{Statistical Analysis}

Data was analyzed using SYSTAT 10 (SPSS). Statistical significance was determined by analysis of variance (ANOVA) followed by post hoc, Dunnett's test, independent $t$-test or by one sample $t$-test where specified. Significant differences are indicated by $* \mathrm{p}<0.05, * * \mathrm{p}<0.01$, and $* * * \mathrm{p}<0.001$.

\section{Results}

\subsection{Preparation and Characterization of Hydrogel Fibers}

We have developed a facile approach for the fabrication of chemically cross-linked alginate fibers loaded with silver nanoparticles (Supporting Figure S1). In this method, the fibers are prepared by wet spinning method in which an alginate solution is injected in a coagulation bath containing calcium ions through a spinneret. Divalent calcium ions cause the gelation of alginate which precipitates at the bottom of the coagulation bath in the form of fibers. Figure 1a shows the representative SEM micrograph of a dried alginate fiber. The diameter of the fiber in the dry state was found to be ca. $100 \mu \mathrm{m}$. In the next step, the alginate gel fibers were further cross-linked with glutaraldehyde to ensure their chemical stability in a wide range of $\mathrm{pH}$ values and salt concentrations. The reaction between hydroxyl groups of sodium alginate and carbonyl groups of glutaraldehyde led to the formation of acetal linkages as confirmed with FTIR spectra (Supporting Figure S2). The chemically cross-linked fibers were robust enough to serve as chemical reactors for the synthesis of silver nanoparticles as discussed below. However, this was not the case for calcium cross-linked alginate fibers which lost the integrity after the treatment with solutions containing silver salt and reducing agent.

\subsection{Superabsorbent Properties of Chemically Cross-Linked Alginate Fibers}

During the cross-linking step calcium, counter-ions bound to carboxyl groups of alginate were partially exchanged with protons of the catalyst $(\mathrm{HCl})$ to form ionizable $\mathrm{COOH}$-groups, which made alginate fibers highly water-swellable at $\mathrm{pH}$ values above the $\mathrm{p} K_{\mathrm{a}}$ of alginic acid. Such fibers exhibit superabsorbent properties, i.e. they could absorb more than 10 times of their own weight in water. ${ }^{[38]}$ In particular, the gravimetric measurements showed that the chemically cross-linked alginate fibers experienced dramatic 20-fold swelling compared to the restricted 3-fold swelling of the ionically cross-linked counterparts (Supporting Figure S3).

\subsection{Synthesis of Silver Nanoparticles in the Alginate Fibers}

The chemically cross-linked alginate network was found to be an appropriate medium for the synthesis of silver nanoparticles. In this method, alginate fibers were first loaded with silver ions by ion exchange and then reduced to metallic silver. The fibers with the reduced silver have the characteristic yellowish hue. The formation of metallic nanoparticles was confirmed by UV-vis spectroscope, TEM, and AFM imaging techniques. The alginate network was enzymatically degraded with alginate 


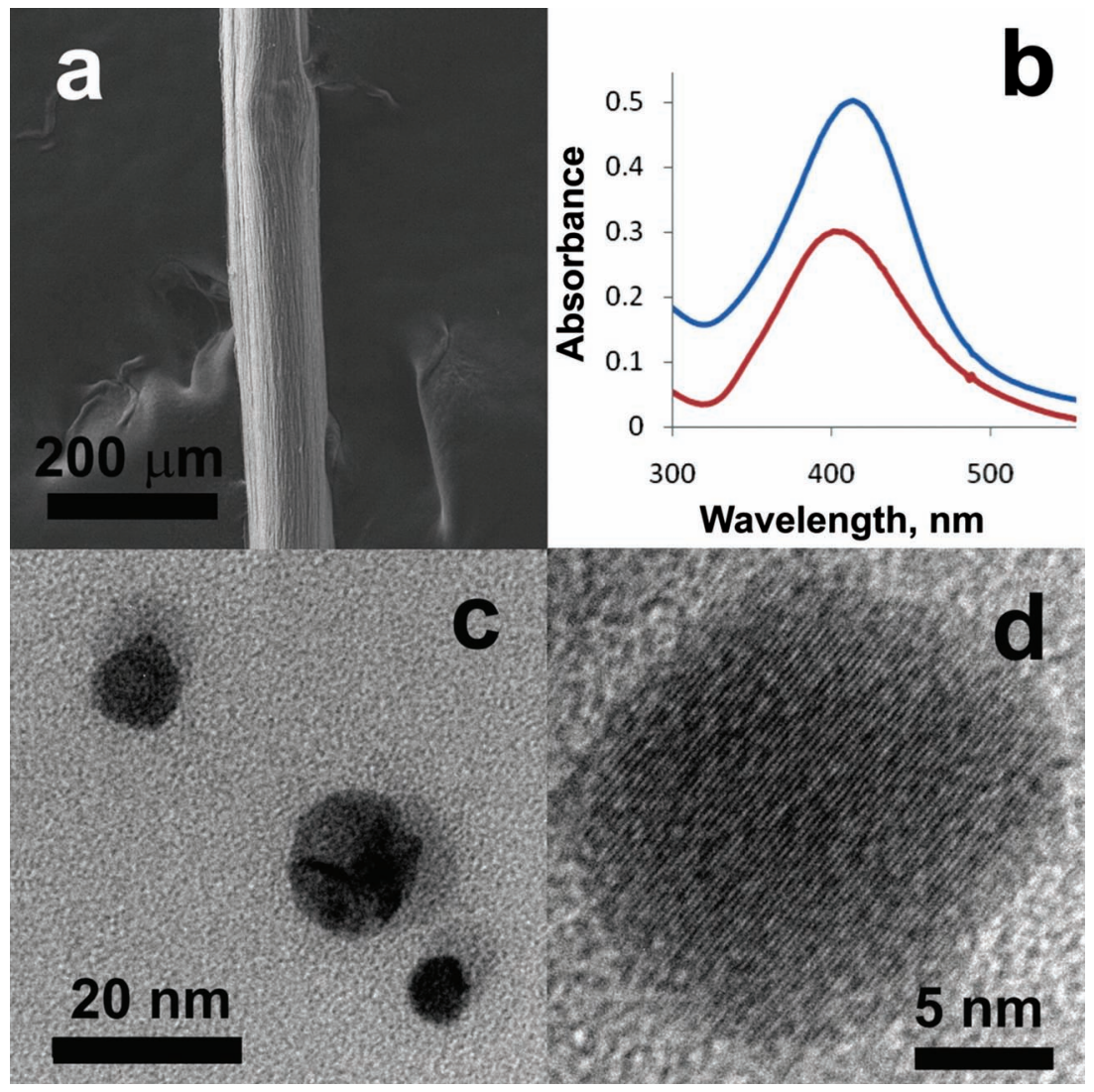

Figure 1. Characterisation of silver nanoparticles and alginate fibers. A) The SEM image of the dried chemically cross-linked alginate fiber. B) The UV-vis spectra of silver nanoparticles synthesized in the chemically cross-linked network of alginate fibers (blue line) and, as a reference experiment, in an aqueous medium (red line) under the same conditions: $0.05 \mathrm{mM} \mathrm{AgNO}_{3}$ and $0.07 \mathrm{mM} \mathrm{NaBH}_{4}$. C) and D) Characteristic TEM images of mono- and polycrystalline silver nanoparticles extracted from the enzymatically degraded fibers; the lattice fringes of a monocrystalline silver nanoparticle can be seen on the high-resolution TEM image.

lyase, and the reduced silver was released into an aqueous medium and deposited onto a suitable substrate (TEM grid or silicon wafer) for TEM and AFM analysis. The UV-vis transmission spectrum of particle dispersion (Figure 1b) exhibits a pronounced extinction band at $412 \mathrm{~nm}$ that arises from the localized surface plasmon resonance (LSPR) phenomenon characteristic of silver nanoparticles. Furthermore, the highresolution TEM images (Figure 1c and d) show that the reduced silver consists of mono- and polycrystalline particles with the average size of $11.5 \pm 5.9 \mathrm{~nm}$. The obtained value is close to the average particle size measured by AFM for Ag nanoparticles deposited on a Si substrate $(9.7 \pm 4.0 \mathrm{~nm}$, Supporting Figure S4).

The amount of the reduced silver was determined with SEMEDS and AAS techniques. SEM-EDS measurements carried out on the fiber surface and in cross-sectional planes of the cryogenically fractured fibers (Supporting Figure S5) show some excess of silver on the fiber surface compared to the fiber interior (about one third higher for the sample loaded from the $0.05 \mathrm{mM}$ $\mathrm{AgNO}_{3}$ solution). Nevertheless, the silver amount on the fiber surface is small compared to that in the fiber bulk and hence the total metal amount is very close to the amount found in the fiber interior. The total amount of the reduced silver in the fiber samples determined with AAS is 0.383 wt.\% in the case fibers loaded in $0.05 \mathrm{mM} \mathrm{AgNO}$ solution and $0.033 \mathrm{wt} . \%$ in the case of fibers loaded in $0.005 \mathrm{mM} \mathrm{AgNO}_{3}$ solution.

\subsection{Biocompatibility of Silver Nanoparticles}

We first investigated the potential cytotoxicity of the silver nanoparticles in mouse 3T3 fibroblast cells. In these experiments we used silver nanoparticles $(\mathrm{d}=11 \mathrm{~nm})$. Mitochondrial metabolic activity, associated with cell viability, was assessed using the MTT assay following exposure to silver nanoparticles and $\mathrm{AgNO}_{3}$ in equal concentrations with respect to silver $(0-5 \mu \mathrm{M})$ for 48 hours. There was no significant reduction in metabolic activity of cells treated with AgNPs, while significant toxicity of $\mathrm{AgNO}_{3}$ was observed at equivalent silver concentrations (Supporting Figure S6). Further investigation revealed that $\mathrm{AgNO}_{3}$ toxicity is likely caused by a reduction in mitochondrial membrane potential, as observed with tetramethylrosamine (TMRE), a fluorescent probe which is rapidly internalized into the cell and is localized to healthy mitochondria due to a strong positive charge (Supporting Figure S7). ${ }^{[39]}$ Mitochondrial membrane potential was maintained following exposure to silver nanoparticles. These results suggest that silver nanoparticles are biocompatible and well tolerated in 3T3 fibroblast cells at all concentrations tested. All subsequent experiments use concentrations of silver nanoparticles within this range and are considered to be non-toxic.

\subsection{Effect of Silver Nanoparticles on Fibroblast Cells Migration to the Wound Area}

Next we chose to investigate the effect of silver nanoparticles on the migration of fibroblasts in cell culture. The scratch assay is a well characterized method to measure cell migration in vitro. ${ }^{40]}$ Mouse fibroblast cells (3T3) are first grown to confluency, and then a scratch is made across the plate. The ability of migrating cells to repopulate the scratch area over time (24-48 hours) was measured. Cells were treated with increasing concentrations of silver nanoparticles $(\mathrm{d}=11 \mathrm{~nm}, 0-5 \mu \mathrm{M})$, FGF $(0.291 \mu \mathrm{M})$ as positive control and MitC $(15 \mu \mathrm{M})$ as negative control. ${ }^{[41]}$ The scratch area of cells treated with increasing concentrations of silver nanoparticles $(0-5 \mu \mathrm{M})$ alone or in combination with FGF appeared to have a greater number of fibroblasts present within the scratch region when compared to untreated cells (CTL; Figure 2A). Semi-quantification of fibroblast cell number within the scratch area, revealed a significant increase in the number of migratory cells when exposed to increasing concentration of silver nanoparticles $(0.31 \mu \mathrm{M}$ AgNP: $1.4+/-0.1$ fold increase; 

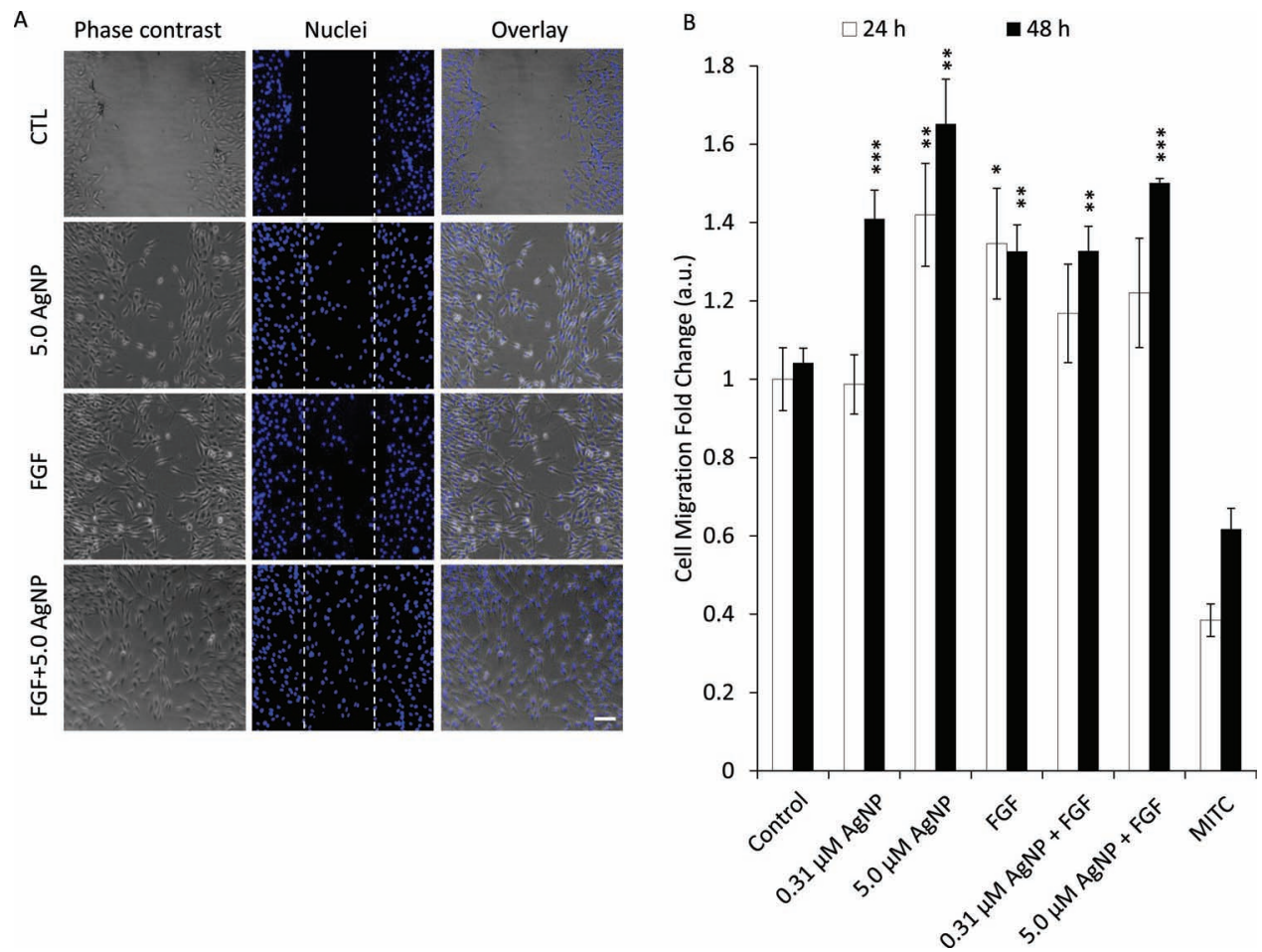

Figure 2. Effect of silver nanoparticles on fibroblast cell migration. A) Fibroblast cell migration was measured using the in vitro scratch assay. Monolayer fibroblast cells were scratched with a pipette tip and treated with silver nanoparticles $(0.31 \mu \mathrm{M}$ and $5 \mu \mathrm{M}$ with respect to silver). Control treatments were i) fibroblast growth factor (FGF, $0.291 \mu \mathrm{M})$ and ii) mitomycin C (MitC, $15 \mu \mathrm{M})$ for 24-48 hours. Cells were then washed, fixed and nuclei were stained with Hoechst $33258(10 \mu \mathrm{M}, 10 \mathrm{~min})$ and fluorescent images of the scratch and surrounding area were acquired. Representative fluorescent microscopy images showing fibroblast cell migration after 48 hours are shown. The scratch area is denoted by dashed white lines. Scale bar $=20 \mu m$. B) The number of migrating cells to the vicinity of the scratch area was determined from the fluorescent images using image J software and expressed as the fold change in cell migration with respect to the control (set to 1). The data is presented as the mean \pm SEM obtained from at least three independent experiments performed in triplicates. Statistically significant differences from the untreated control were tested by Dunnett's test and are indicated by $\mathrm{p} *<0.05, \mathrm{p} * *<0.01, \mathrm{p} * * *<0.0$

$0.5 \mu \mathrm{M}$ AgNP: $1.62+/-0.1$ fold increase) 48 hours following the application of the scratch alone or in combination with FGF (Figure 2B). There is a dose dependent increase in cell migration following exposure to silver nanoparticles (Supporting Figure S8). These observations suggest that wound healing is accelerated by the concentration of silver.

\subsection{Effect of Alginate Fibers Loaded with Silver Nanoparticles on the Inflammatory Phase of Wound Healing}

To better understand the action of alginate fibers and silver nanoparticles in wound healing, we took advantage of a well characterized in vivo incisional wound model. Hairless SKH-1 mice were separated into five different treatment groups: i) untreated, ii) unloaded fiber, iii) AgNP loaded fiber $(0.383 \%$ Ag), iv) AgNP loaded fiber (0.033\% Ag) and v) silver nanoparticles alone $(0.383 \% \mathrm{Ag})$. Once anesthetized, $2 \mathrm{~cm}$ long incisional wounds were applied surgically to the mice, as described in Methods. Immunohistochemistry was used to stain for neutrophils and macrophages (inflammatory mediators of wound healing), from animals sacrificed on postoperative day 2. Silver nanoparticle loaded alginate fibers and silver nanoparticle treatment alone significantly reduced neutrophil infiltration at local wound site (Figure 3a and b). Treatment with silver nanoparticles alone resulted in a significant decrease in macrophage infiltration. Indeed, AgNPs alone are more effective than AgNP loaded fibers in decreasing macrophage/neutrophil numbers. These results suggest a diminished inflammatory response following exposure to silver nanoparticles.

\subsection{Healing Promotion and Cosmetic Effects}

We next compared the appearance of the healed wounds by making histological sections from animals on postoperative day 10 . We found that wounds in the silver nanoparticle alone group showed the most resemblance to normal skin. The worst cosmetic appearance was observed in the untreated group. Under histological evaluation, healed wounds from the silver nanoparticle group showed a significantly increased epidermal thickness (Figure 4A). These observations further confirmed that silver nanoparticles accelerate wound closure and improve wound healing quality. Taken together with the in vitro data, our results suggest that silver nanoparticles may promote fibroblast migration, reduce the inflammatory cell recruitment to the wound and result in improved wound healing. 


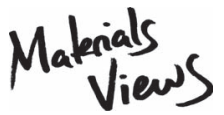

www.MaterialsViews.com

www.advhealthmat.de
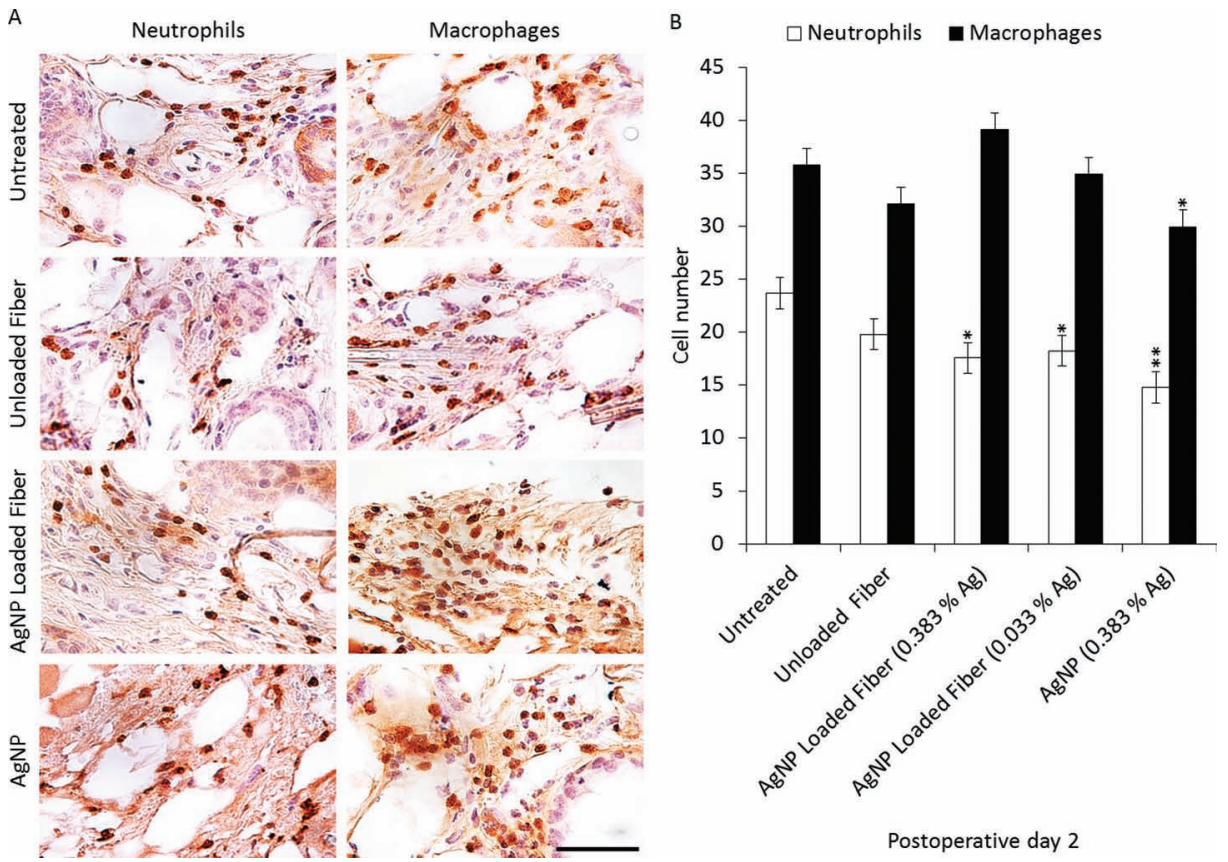

Figure 3. Decreased neutrophil infiltration at local wound site by alginate fibers loaded with silver nanoparticles. Incisional skin wounds were created on the dorsum of hairless SKH-1 mice and they were divided into 5 groups: i) treated with unloaded alginate fibers, ii) silver nanoparticle loaded alginate fibers ( $0.383 \mathrm{wt} . \% \mathrm{Ag})$, iii) silver nanoparticle loaded alginate fibers $(0.033 \mathrm{wt} . \% \mathrm{Ag})$, iv) silver nanoparticles alone and v) untreated control. A) Immunohistochemical staining for neutrophils and macrophages in each experimental group on day 2 following the incisional wound injury. Positive staining is indicated by distinct brown spots. Scale bars $=200 \mu \mathrm{m}$. B) Semi-quantification of neutrophil and macrophage cell number from representative microscopy images. The data is presented as the mean \pm SEM obtained from at least three independent experiments performed. Statistically significant differences form the untreated control were tested with Dunnett's test and are indicated by $* p<0.05$ and $* * p<0.01$
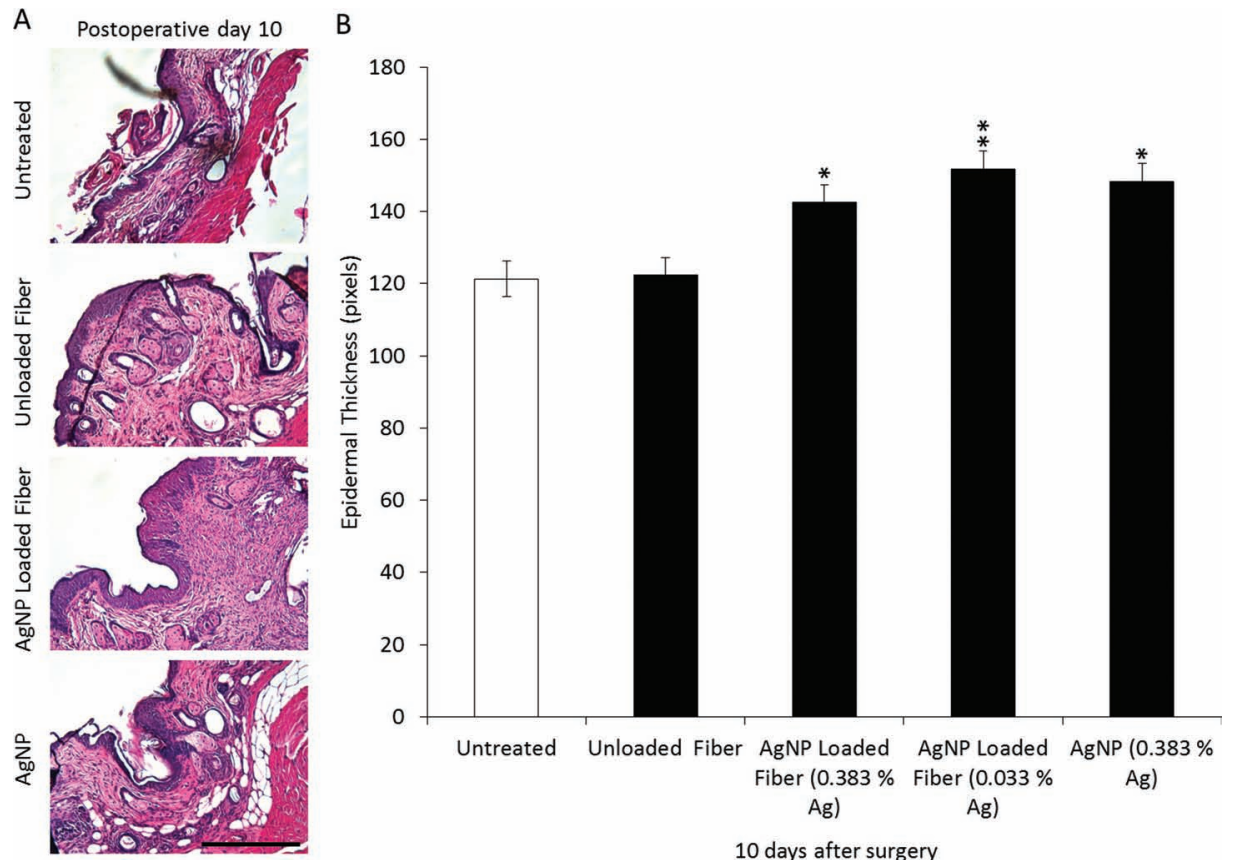

Figure 4. Increased epidermal thickness in wound tissue exposed to alginate fibers loaded with silver nanoparticles. Incisional skin wounds were created on the dorsum of hairless SKH-1 mice and they were divided into 5 groups and were treated with either unloaded alginate fibers, silver nanoparticle loaded alginate fibers ( $0.383 \mathrm{wt} . \% \mathrm{Ag}$ and $0.033 \mathrm{wt} . \% \mathrm{Ag})$, silver nanoparticles alone or remained untreated. A) Haematoxylin and eosin (H\&E) histological staining of wounded skin in each experimental group on day 10 following incisional wound injury. Scale bars $=500 \mu m$. B) Semiquantification of epidermal thickness from representative microscopy images. The data is presented as the mean \pm SEM obtained from at least three independent experiments. Statistically significant differences from the untreated control were tested with Dunnett's test and are indicated by $* \mathrm{p}<$ 0.05 and $* * \mathrm{p}<0.01$. 


\section{Discussion}

The results presented in the current study show that silver based wound management materials can be easily prepared by incorporating silver nanoparticles on to alginate fibers, which promote healing in an in vivo incisional wound model. Our results, together with the findings of several other studies, suggest that silver and silver related compounds are highly effective in wound management. ${ }^{[42]}$ Despite being used in low concentrations, there are major toxicity concerns with silver based materials such as silver nitrate. ${ }^{[4]}$ Skin exposure to high concentrations of silver can result in the subcutaneous formation of colloidal silver or silver salts, known as argyria. Silver toxicity appears to be associated with the impairment of mitochondrial function, due to increased mitochondrial membrane permeability. ${ }^{[4]}$ These findings are further supported by our studies involving TMRE staining. Silver nanoparticles are significantly less toxic than silver nitrate. This is in part due to the slow release of silver ions over time from the silver nanoparticles. Our results are consistent with observations reported in the literature and suggests a limited toxicity of nanosilver, related to the unique silver species released (e.g., $\mathrm{Ag}^{0}$ ), that is different from the indiscriminate activity of $\mathrm{Ag}^{+}$ions from silver nitrate. Apoptotic activity of nanostructured silver is discriminatory towards dermal cells, where as $\mathrm{AgNO}_{3}$ apoptotic activity is indiscriminate and likely induced in all cell types at the tissue surface, including keratinocytes. ${ }^{[45]}$

In order to reduce toxicity, silver can be incorporated and delivered to the wound site using an appropriate carrier, such as alginate based materials. Traditionally, silver ions from silver nitrate $\left(\mathrm{AgNO}_{3}\right)$ solution are exchanged with calcium ions from alginate fibers, resulting in the formation of calcium alginate fiber containing silver ions. ${ }^{[33]}$ As previously discussed, the substitution of $\mathrm{Ca}^{2+}$-cross-links with $\mathrm{Ag}^{+}$-ions may compromise the mechanical stability of alginate fibers. Fiber stability is especially important in the inflamed wound microenvironment, which is characterized by increased acidity and temperature. We addressed the stability issue in this study through the two-step crosslinking process in which ionic cross-links involving carboxylic groups are substituted with covalent ones involving hydroxyl groups, hence liberating the carboxyl groups for binding $\mathrm{Ag}^{+}$-ions. Furthermore, the covalent cross-linking makes the fibers sufficiently robust to withstand the aggressive conditions associated with the chemical reduction of $\mathrm{Ag}^{+}$-ions to nano-sized silver particles occurring in the alginate matrix. By incorporating silver nanoparticles into alginate fibers, it is expected that a highly absorbent and stable wound dressing with good antimicrobial properties can be obtained, while minimizing silver related toxicity.

Alginate fibers provide an attractive scaffolding for the delivery of silver nanoparticles and result in the sustained release of silver ions to the wound area by three primary mechanisms: i) ion exchange between the silver ions released from the nanoparticles attached to the fiber and sodium and calcium ions in the wound exudate, ii) silver ions are chelated by proteins present in the wound, iii) silver nanoparticles embedded into the alginate detach over time (due to the biodegradation of the alginate fibers) and enter the wound exudate. ${ }^{[3]}$ Silver nanoparticles in an aqueous solution can disaggregate into clusters containing two to eight silver atoms. ${ }^{[46]}$ These clusters are believed to be responsible for the anti-inflammatory activity. ${ }^{[45]}$ Liberated silver clusters and ions can then easily bind to chlorides and proteins. Indeed, silver is released more quickly into human serum than normal saline, suggesting high silver binding capacity of the protein components in the wound exudates. ${ }^{[3]}$ This permits for the transport of silver ions to areas outside the immediate site of administration.

While metallic silver $\left(\mathrm{Ag}^{0}\right)$ is relatively inactive, silver ions $\left(\mathrm{Ag}^{+}\right)$exert antibacterial activity primarily by interfering with the respiratory chain of microbes and binding to bacterial DNA, inhibiting DNA replication. ${ }^{[47]}$ Additionally, nanosilver has been shown to possess anti-inflammatory properties in infectious wounds, by modulating cytokine production. ${ }^{[48]}$ This is believed to occur primarily through the reduced production of cytokines, including TNF- $\alpha$ and IL-8, however the precise mechanisms remain unclear. ${ }^{[49]}$ Other noble metals have anti-inflammatory activity, including titanium, copper and gold. ${ }^{[50,51]}$ Previous studies have shown that $\mathrm{Au}^{+}$is effective in treating arthritis symptoms through oxidation/reduction reactions that produces $\mathrm{Au}^{3+}$ and $\mathrm{Au}^{0}{ }^{[52]} \mathrm{Au}^{0}$ nanoparticles have been shown to inhibit the activation of IL- 6 and TNF- $\alpha .^{[52]}$ As with silver, $\mathrm{Au}^{0}$ atoms do not exist in solution, suggesting that both active $\mathrm{Au}$ and $\mathrm{Ag}$ species form clusters.

In the current study, the therapeutic efficacy of nanosilver loaded alginate fibers in wound healing was assessed using the scratch assay as an in vitro model system to mimic an incisional wound in skin cells. Fibroblast cells are a commonly used representative model of skin cells when investigating cell migration/proliferation. Our results from in vitro model system show that silver nanoparticle exposure is non-toxic and increases the number of fibroblasts counted in the vicinity of the wound after 48 hours compared to untreated control. However, treatment with silver nanoparticles did not increase fibroblast migration above that induced by FGF alone, or in combination treatment with FGF. These finding raise the possibility that silver nanoparticles may function through a similar mechanism, as we observed no synergy between FGF and silver loaded alginate fiber treatment. Alternatively, cell migration may be a saturable effect at very low concentrations of FGF. This remains to be experimentally determined. Growth factors such as FGF and VEGF are well characterized promoters of endothelial cell proliferation and the physical reorganization of endothelial cells. ${ }^{\text {[53] }}$ In addition, FGF plays a role in wound healing through the stimulation of angiogenesis and the proliferation of fibroblasts that give rise to granulation tissue, which fills up a wound area early in the wound-healing process. ${ }^{[54]}$

The presence of an increased number of fibroblasts in the wound area following the scratch could be accounted by enhanced fibroblast migration and/or proliferation. Nanosilver has been shown to increase the rate of wound closure through the promotion of proliferation and migration of keratinocytes and can drive differentiation of fibroblasts into myofibroblasts, thereby promoting wound contraction. ${ }^{[55]}$ Indeed, silver nanoparticles can drive the differentiation of resident fibroblasts into myofibroblasts in the mid-to-late phases of wound healing. ${ }^{[55]}$ We found that the migration of fibroblasts was facilitated by increased concentrations of silver nanoparticles, suggesting that this effect likely depends on the concentration of silver 
ions in the wound area. Fibroblast to myofibroblast transition induces expression of contractile elements in fibroblasts that promotes migration. ${ }^{[56]}$ Differentiation of fibroblasts into myofibroblasts can be understood as a two-step process. First, to re-populate damaged tissues, fibroblasts acquire a migratory phenotype and invade the wound area. This phenotypic change occurs in response to changes in the composition, organization, and mechanical property of the extracellular matrix (ECM) and to cytokines locally released by inflammatory and resident cells. Second, with increasing stress in the ECM resulting from their own remodeling activity, proto-myofibroblasts further develop into differentiated myofibroblasts by expressing $a$-smooth muscle actin ( $a$-SMA), the most widely used myofibroblast marker. Expression of $a$-SMA is precisely controlled by the complementary action of growth factors like transforming growth factor (TGF-b), which is believed be down regulated by nanosilver. ${ }^{[45]}$

We observed clear morphological changes during inflammation and re-epithelialization processes. From the histological evaluations, we noticed a faster wound closure in mice treated with AgNPs and silver loaded fibers as compared with control. Our results confirmed previously established effects of AgNPs on wound healing namely: accelerated re-epithelialization, enhanced migration of fibroblasts and reduced neutrophil and macrophage infiltration at the wound site. In previous studies, AgNPs have shown to improve tensile properties and better fibril alignments in repaired skin as they are responsible for regulating deposition of collagen in the wound healing process. ${ }^{[57]}$ This can be confirmed by inspecting collagen organization using masson's trichrome or picrosirius red staining. The effect of reduced inflammation was stronger for silver nanoparticles directly incorporated into the wound as compared to silver loaded alginate fibers. That is likely due to a delayed release of silver ions from the fibers in the initial stages of the wound healing. However, in the later stages of the wound contraction we observed less difference between the fibers and reference silver nanoparticles because of biodegradation of alginate in the later stage. The in vivo model system used in this study provided a clean and infection-free wound model. While the presence of the silver loaded alginate fibers may reduce inflammation and therefore the capacity to fight off infections, in the event that a treated wound is infected, the presence of the silver ions and their anti-microbial properties might off-set this effect and still heal more effectively than an untreated wound.

Several studies have demonstrated that apoptosis is involved in the elimination of inflammatory cells from inflamed tissues, and that nanosilver treatments are capable of inducing apoptosis specific to inflammatory cells. ${ }^{[58,59]}$ Studies from the Burrell group suggest that silver ions released from nanosilver do not produce anti-inflammatory activity solely by direct interaction with pro-inflammatory cells, rather, nanosilver can impact wound healing remotely from the site of application. ${ }^{[60]}$ Interestingly, their findings show a reduced potency, but not delayed response to the remote nanosilver treatment which suggests that changes to biological signals are being produced at the site of application, leading to a rapidly propagating biological cascade effect, rather than a direct silver interaction with inflammatory cells. The potential biological cascade hypothesis is controversial and requires further investigation.
Taken together, these findings further extend our current knowledge of AgNPs in biological and cellular events and also have significant implications for the treatment of wounds in the clinical setting. Other experiments are required to further investigate mechanisms by which silver nanoparticles accelerate wound healing.

\section{Conclusions}

In this work, we show that silver nanoparticles improve wound healing. Chemically cross-linked alginate fibers provide a robust platform of delivery silver nanoparticles into the wound area. The alginate fibers loaded with silver nanoparticles reduce the inflammatory phase and increase epidermal thickness, improving the overall quality and speed of healing. Several mechanisms of action for the effects of the silver nanoparticle have been discussed, however further in depth studies are required to investigate the effect of particle size, morphology, surface properties, and the associated signal transduction mechanisms mediating wound healing processes.

\section{Supporting Information}

Supporting Information is available from the Wiley Online Library or from the author.

\section{Acknowledgements}

The authors would like to acknowledge Eliza Hutter and Emilia Kowalski for assistance with image analysis. This material is based upon work supported by the U. S. Army Research Laboratory and the U. S. Army Research Office under grant W911NF-05-1-0339 and the Canadian Institute of Health Research (CIHR). Note: The authors declare no competing financial interests.

Received: March 9, 2012

Revised: May 2, 2012

Published online: July 12, 2012

[1] E. H. Epstein, New. Engl. J. Med. 1999, 341, 9.

[2] T. Kondo, Y. Ishida, Forensic Sci. Int. 2010, 203, 93.

[3] L. G. Ovington, Home Healthcare Nurse 2002, 20, 652.

[4] S. Petrulyte, Dan. Med. Bull. 2008, 55, 72.

[5] Y. Qin, Polym. Int. 2008, 57, 10.

[6] S. S. Silva, J. F. Mano, R. L. Reis, Crit. Rev. Biotechnol. 2010, 30, 200.

[7] R. L. Coleman, G. Lawrie, L. K. Lambert, M. Whittaker, K. S. Jack, L. Grondahl, Biomacromolecules 2011, 12, 889.

[8] A. Smelcerovic, Z. Knezevic-Jugovic, Z. Petronijevic, Curr. Pharm. Des. 2008, 14, 3168.

[9] M. D. Groenewold, A. J. Gribnau, D. T. Ubbink, BMC Surg. 2011, 11, 15.

[10] J. G. Thomas, W. Slone, S. Linton, T. Okel, L. Corum, S. L. Percival, J. Wound Care 2011, 20, 124.

[11] S. Thomas, J. Wound Care 2000, 9, 56.

[12] Y. Qin, J. Appl. Polym. Sci. 2003, 91, 5.

[13] H. H. Tonnesen, J. Karlsen, Drug Dev. Ind. Pharm. 2002, 28, 621. 
[14] W. J. Cho, S. H. Oh, J. H. Lee, J. Biomater. Sci. Polym. Ed. 2010, 21, 701.

[15] M. Walker, J. A. Hobot, G. R. Newman, P. G. Bowler, Biomaterials 2003, 24, 883.

[16] T. Coviello, P. Matricardi, F. Alhaique, Expert Opin. Drug Delivery 2006, 3, 395.

[17] Q. Zeng, W. Chen, Biomaterials 2010, 31, 5772.

[18] L. Bian, D. Y. Zhai, E. Tous, R. Rai, R. L. Mauck, J. A. Burdick, Biomaterials 2011, 32, 6425 .

[19] P. W. Henderson, S. P. Singh, D. D. Krijgh, M. Yamamoto, D. C. Rafii, J. J. Sung, S. Rafii, S. Y. Rabbany, J. A. Spector, Spectorm Wound Repair Regen. 2011, 19, 420.

[20] T. J. Wlodkowski, H. S. Rosenkranz, Lancet 1973, 2, 739.

[21] H. Q. Yin, R. Langford, R. E. Burrell, J. Burn Care Rehabil. 1999, 20, 195.

[22] S. Thomas, P. McCubbin, J. Wound Care 2003, 12, 305.

[23] C. Elliott, Br. J. Nurs. 2010, 19, 32.

[24] S. Hussain, C. Ferguson, Emerg. Med. J. 2006, 23, 929.

[25] S. L. Percival, J. G. Thomas, W. Slone, S. Linton, L. Corum, T. Okel, Wound Repair Regen. 2011, 19, 767.

[26] J. B. Wright, K. Lam, R. E. Burrell, Am. J. Infect. Control 1998, 26, 572.

[27] X. Chen, H. J. Schluesener, Toxicol. Lett. 2008, 176, 1.

[28] N. Lubick, Environ. Sci. Technol. 2008, 42, 8617.

[29] J. B. Wright, K. Lam, A. G. Buret, M. E. Olson, R. E. Burrell, Wound Repair Regen. 2002, 10, 141.

[30] R. Foldbjerg, P. Olesen, M. Hougaard, D. A. Dang, H. J. Hoffmann, H. Autrup, Toxicol. Lett. 2009, 190, 156.

[31] K. Chaloupka, Y. Malam, A. M. Seifalian, Trends Biotechnol. 2010, 28, 580.

[32] R. Cortivo, V. Vindigni, L. lacobellis, G. Abatangelo, P. Pinton, B. Zavan, Nanomedicine 2010, 5, 641.

[33] Y. Qin, Int. Wound J. 2005, 2, 172.

[34] A. A. Panteleyev, R. Paus, W. Ahmad, J. P. Sundberg, A. M. Christiano, Exp. Dermatol. 1998, 7, 249.

[35] K. Tomihata, Y. Ikada, J. Polym. Sci. Part A: Polym. Chem. 1997, 35, 3553.

[36] T. Mosmann, J. Immunol. Methods. 1983, 1-2, 55.

[37] H. P. Ehrlich, T. K. Hunt, Ann. Surg. 1969, 170, 203.
[38] M. J. Zohuriaan-Mehr, H. Omidian, S. Doroudiani, K. Kabiri, J. Mater. Sci. 2010, 45, 5711.

[39] H. J. Eom, J. Choi, Environ. Sci. Technol. 2010, 21, 8337.

[40] C. C. Liang, A. Y. Park, J. L. Guan, Nat. Protoc. 2007, 2, 329.

[41] Y. Abe, K. Inagaki, A. Fujiwara, K. Kuriyama, Eur. J. Pharmacol. 2000, 2, 213.

[42] B. S. Atiyeh, M. Costagliola, S. N. Hayek, S. A. Dibo, Burns 2007, 33, 139.

[43] S. D. Humphreys, P. A. Routledge, Adverse Drug React. Toxicol. Rev. 1998, 17, 115

[44] J. S. Teodoro, A. M. Simoes, F. V. Duarte, A. P. Rolo, R. C. Murdoch, S. M. Hussain, C. M. Palmeira, Toxicol. In Vitro 2011, 25, 664.

[45] P. L. Nadworny, J. Wang, E. E. Tredget, R. E. Burrell, Nanomedicine 2008, 4, 241.

[46] F. R. F. Fan, A. J. Bard, J. Phys. Chem. B 2002, 106, 8.

[47] Q. L Feng, J. Wu, G. Q. Chen, F. Z. Cui, T. N. Kim, J. O. Kim, J. Biomed. Mater. Res. 2000, 52, 662.

[48] J. Tian, K. K. Wong, C. M. Ho, C. N. Lok, W. Y. Yu, C. M. Che, J. F. Chiu, P. K. H. Tam, ChemMedChem 2007, 2, 129.

[49] P. L. Nadworny, J. Wang, E. E. Tredget, R. E. Burrell, J. Inflamm. 2010, 7, 1.

[50] Y. C. Paquay, J. E. de Ruijter, J. P. van der Waerden, J. A. Jansen, Biomaterials 1997, 18, 161.

[51] L. Thunus, J. F. Dauphin, G. Moiny, C. Deby, G. Deby-Dupont, Analyst 1995, 120, 967.

[52] G. E. Abraham, P. B. Himmel, J. Nutr. Environ. Med. 1997, 7, 295.

[53] J. Suehiro, T. Hamakubo, T. Kodama, W. C. Aird, T. Minami, Blood 2010, 115, 2520.

[54] J. G. Lee, E. P. Kay, Invest. Ophthalmol. Vis. Sci. 2006, 47, 1376.

[55] X. Liu, P. Y. Lee, C. M. Ho, V. C. Lui, Y. Chen, C. M. Che, P. K. Tam, K. K Wong, ChemMedChem 2010, 5, 468.

[56] B. Hinz, J. Invest. Dermatol. 2007, 127, 526.

[57] K. H. Kwan, X. Liu, M. K. To, K. W. Yeung, C. M. Ho, K. K. Wong, Nanomedicine 2011, 7, 497.

[58] D. G. Greenhalgh, Int. J. Biochem. Cell Biol. 1998, 30, 1019.

[59] C. N. Serhan, J. Savill, Nat. Immunol. 2005, 6, 1191.

[60] P. L. Nadworny, B. K. Landry, J. Wang, E. E. Tredget, R. E. Burrell, Wound Repair Regen. 2010, 18, 254. 